\title{
Identification of Candida Species Circulating in Dakar using Multiplex Nested PCR
}

\author{
Badiane $\mathrm{AS}^{1,2 *}$, Diongue $\mathbf{K}^{1,2}$, Sène $\mathrm{A}^{2}$, Cheikh Seck $\mathbf{M}^{1,2}$, Alpha Diallo $\mathbf{M}^{2}$, \\ Ndiaye $\mathrm{M}^{1,2}$ and Ndiaye $\mathrm{D}^{1,2}$ \\ ${ }^{1}$ Laboratory of Parasitologie-Mycology, Faculty of Medicine, Universite Cheikh Anta Diop, \\ Senegal \\ ${ }^{2}$ Laboratoire de Parasitologie-Mycologie, Hospital Aristide Le Dantec, Senegal
}

*Corresponding author: Aida Badiane, Laboratory of Parasitologie-Mycology, Faculty of Medicine, Universite Cheikh Anta Diop, Dakar, Senegal, Tel: 00221776555527; Email: asbadiane@gmail.com

\section{Abstract}

Background: In most developing countries, laboratory identification of Fungi belonging to the genus Candida is often problematic. The germ test tube known as Blastese test, which is the most used has the limitation that it only differentiates the C. albicans complex from other Candida complexes. Not only it is important to know the involvement of other species in the occurrence of fungal infections, but also the difference in sensitivity to the azoles used in the treatment of these fungal infections makes it necessary to identify the Fungi at the species level. The objective of this study was to identify by multiplex nested PCR the Candida species isolated in the laboratory of Parasitology and Mycology of Aristide Le Dantec Hospital, Senegal.

Methodology: Candida isolates were obtained from patients who were admitted to the laboratory of Parasitology and Mycology with suspected fungal infections. After identification by conventional methods, isolates were preserved in a storage medium and kept at $-20^{\circ} \mathrm{C}$. Species identification was performed by nested PCR.

Results: A total of 42 yeast isolates were collected after culture on Sabouraud medium, among which 10 (24\%) from vaginal swabs and 32 (76\%) from patients suffering from superficial mycoses (skin and nails). C. albicans represented 68\% and nonCandida albicans (NAC) species 32\%. The distribution of the NAC species was as follows: C. parapsilosis (group I and II) $17 \%$ (8), C. tropicalis 8.5\% (4), C. kefyr 4.25\% (2) and C. lusitaniae 2.15\% (1). Mixed infection with two species represented 17.39\% of the isolates.

Conclusion: This study shows that other Candida species are common in Dakar, although their identification is not routinely performed in most laboratories. Thus, it is necessary to improve the technical facilities in these diagnostic laboratories.

Keywords: Candida; Multiplex Nested PCR; Identification 


\section{Open Access Journal of Mycology \& Mycological Sciences}

Abbreviations: NAC: Non-albicans Candida; PM: Parasitology-Mycology; PCA: Positive control C. albicans; TNC: Negative control; PCR: Amplification Chain Reaction.

\section{Introduction}

Yeasts belonging to the genus Candida constitute an important part of the normal flora of the digestive tract, the skin and the mucous membranes including the urogenital tract [1]. Therefore, they are responsible for vaginal, skin and nail infections $[2,3]$. In Dakar, vulvovaginal candidiasis represents respectively $24 \%$ in 2006 and $34.8 \%$ in 2008 among women admitted at the CHU Le Dantec of Dakar and $27.2 \%$ in 2015 at the military hospital of Ouakam [4].

Species identification of Candida fungi is essential to reliably initiate an effective treatment since there is a difference in sensitivity of species to azole drugs. For instance, C. glabrata is known to be resistant to fluconazole and $C$. krusei is not overly sensitive to azole drugs.

However, the identification of these yeasts of the genus Candida in developing countries is essentially based on direct microscopic examination; culture followed by a filamentation test to distinguish the C. albicans complex from other complexes grouped under the term "non-albicans" Candida (NAC) species. This conventional diagnosis, which is generally the only available in resource-limited countries, needs to be reinforced by more efficient techniques to give much more accurate and reliable results to better contribute to the proper management of candidiasis. Although in these countries, galleries using auxanogram (assimilation of carbohydrates in an aerobic environment) or zymogram (fermentation in anaerobic environment) are available, the time for reporting results are long and the identification is not always accurate. Similarly, chromogenic media and latex agglutination are sometimes used, but ideally these results should be confirmed by a more reliable technique.

In recent years, molecular biology has led to a diversity of species which requires an improvement in identification techniques for a better management of candidiasis. Molecular tests; such as nested PCR [5] and multiplex PCR [6] have been used for the identification of Candida species. Although C. albicans remains the most represented species in candidiasis, there is an emergence of NAC species in mycosis. Among these species, C. tropicalis, C. glabrata, $C$. krusei, C. parapsilosis, C. kefyr and C. lusitaniae have been the most reported $[3,4,7,8]$. In Senegal, it has been reported that Candida albicans is the predominant species [4,9]. However, the frequency of other Candida species remains unknown. Thus, the molecular techniques such amplification chain reaction (PCR) could be used to identify Candida species in clinical samples. Thus, the objective of this study was to set up in the laboratory a molecular technique to identify Candida species isolated from patients.

\section{Methodology}

Strains were from patients with a prescription for mycological analysis or vaginal sampling who were admitted respectively at the Parasitology-Mycology (LPM) and Bacteriology-Virology laboratories of the Aristide LeDantec hospital in Dakar, Senegal.

The specimens collected were squama for dermatoses, nail debris for onychomycoses and swabs for vaginal infections. Data obtained for the identification of yeast belonging to the genus Candida with classical or phenotypic techniques in the laboratory of Parasitology-Mycology were collected from 2016 to 2019.

\section{Classical Techniques/Phenotypic Identification}

For fungal diagnosis, a direct microscopy examination and a culture was carried out on Sabouraud medium with added chloramphenicol and actidione. The culture was incubated for 24 to 48 hours at 25 to $30^{\circ} \mathrm{C}$ for superficial infections (dermatoses and onychomycoses) and $37^{\circ} \mathrm{C}$ for vaginal samples. The filamentation test was performed to differentiate the albicans complex from other Candida species. These strains were preserved in brain heart broth and glycerol and stored at $-20^{\circ} \mathrm{C}$ until use.

The number of Candida species identified in the LPM was collected and the frequency of Candida albicans and NAC was determined.

\section{Multiplex nested PCR}

DNA was extracted using the QIAamp DNA Mini Kit (Qiagen, Valencia, CA, and USA) according to the manufacturer's instructions. The identification was based on multiplex nested PCR [10], using three primer mixtures named PsI, PsII, and PsIII which identify groups of species based on the size of the specific amplification product obtained for each species (Table 1). The first group included C. albicans, C. parapsilosis (group I), C. orthopsilosis (group II), and C. guilliermondii, the second group is composed of $C$. dubliniensis, C. krusei, C. kefyr, and C. glabrata and the third group identify $C$. tropicalis I, C. tropicalis II and C. lusitaniae. C. parasilopsis and C. tropicalis are divided into at least two genomic types based on DNA sequence analysis of the topoisomerase II gene [11]. 


\begin{tabular}{|c|c|c|c|c|}
\hline Mélange d'amorces & Espèces cibles & Amorce sens & Amorce anti-sens & Taille produit PCR (bp) $^{\mathbf{b}}$ \\
\hline \multirow{4}{*}{ PsI } & C. albicans & CABF59 & CADBR125 & 665 \\
\cline { 2 - 5 } & C. parapsilosis I & CPPIF41 & CPPIR122 & 837 \\
\cline { 2 - 5 } & C. parapsilosis II & CPPIF41 & CPPIIR69 & 310 \\
\cline { 2 - 5 } & C. guilliermondii & CGLF41 & CGLR61 & 205 \\
\hline \multirow{4}{*}{ PsII } & C. dubliniensis & CDBF28 & CDBR110 & 816 \\
\cline { 2 - 5 } & C. krusei & CKSF35 & CKSR57 & 227 \\
\cline { 2 - 5 } & C. kefyr & CKFF35 & CKFR85 & 532 \\
\cline { 2 - 5 } & C. glabrata & CGBF35 & CGBR103 & 674 \\
\hline \multirow{4}{*}{ PsIII } & C. tropicalis I & CTPIF36 & CTPIR68 & 318 \\
\cline { 2 - 5 } & C. tropicalis II & CTPIIF36 & CTPIIR121 & 860 \\
\cline { 2 - 5 } & C. lusitaniae & CLTF39 & CLTR119 & 799 \\
\cline { 2 - 5 } & Y. lipolytica & CLLF35 & CLLR59 & 245 \\
\hline
\end{tabular}

Table 1: Species-specific primer pairs used in each mixture and sizes of each expected PCR product for each species.

For PCR amplification, we first prepared the reaction mixture, which is commonly called Master Mix, just before its use. Thus, for the first amplification for confirmation of the Candida genus, the degenerate sense and anti-sense primers we used: CDF28 and CDR148. For the second amplification, three separate reactions were run for the mix primers PSI, PSII and PSIII.

The first amplification is designed to identify the genus, Candida. The reaction was performed in a final volume of $25 \mu \mathrm{l}$ containing $1 \mu \mathrm{l}$ of DNA, $10 \mu \mathrm{M}$ of CDF28, $10 \mu \mathrm{M}$ of CDR148, $10 \mathrm{mM}$ of dNTPs and $0.125 \mu 1$ DNA Taq polymerase. The second amplification identified the species and was carried out in a final volume of $25 \mu \mathrm{l}$ containing 1 to $5 \mu \mathrm{l}$ of DNA, $10 \mu \mathrm{M}$ of each of the primers for PsI, PsII et PsIII, $10 \mathrm{mM}$ of dNTPs and $0.125 \mu 1$ DNA Taq polymerase. Amplification was performed on T100 Thermal Cycler, Bio-Rad®. The PCR conditions were as follows: Denaturation at $95^{\circ} \mathrm{C}$ for 30 seconds (sec), 40 cycles of $95^{\circ} \mathrm{C}$ for $30 \mathrm{sec}, 62^{\circ} \mathrm{C}$ for $30 \mathrm{sec}$, $74^{\circ} \mathrm{C}$ for $60 \mathrm{sec}$ and $70^{\circ} \mathrm{C}$ for $5 \mathrm{~min}$.

The amplified products were revealed by electrophoretic migration on $2 \%$ agarose gel stained with Ethidium Bromide. The migration was then performed at 90 volts using an electronic cell for $30 \mathrm{~min}$. Visualization was performed under UV light and pictures were taken indicating the size of the amplicons in base pairs (bp) using a molecular weight marker.

\section{Results}

In total, forty-two (42) yeast isolates were collected after culture on Sabouraud medium; 76\% (32/42) were from superficial infections (skin and nails) and 24\% (10/42) from vaginal infections.

\section{Species Identification using Multiplex Nested PCR}

For the amplification with the mixture of PsI primers which are specific for C. albicans, C. parapsilosis I, C. parapsilosis II and C. guilliermondii, only thirteen (13) out of forty-two (42) samples were positive (Figure 1) for C. albicans and 5 for $C$. parapsilosis (type I, type II or both combined).

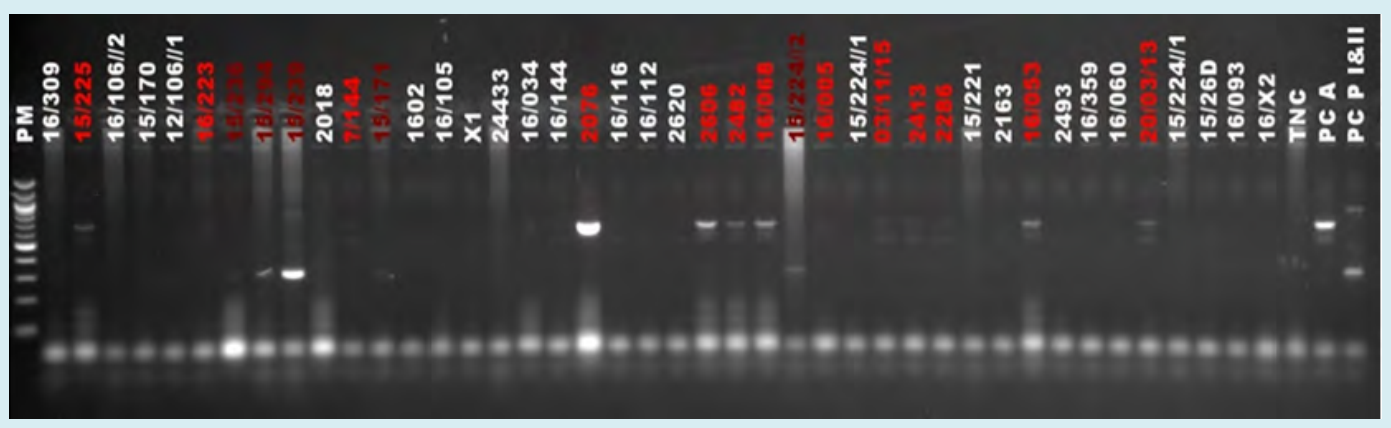

Figure 1: Amplification by multiplex PCR from PsI.

PM: 100 bp molecular weight markers; TNC: Negative control; PCA: Positive control C. albicans; PCP I \& II: Positive control C. parapsilosis type I and type II 


\section{Open Access Journal of Mycology \& Mycological Sciences}

The presumptive negative and low positive samples from this first test were re-run using the same primers, from PsI, but increasing the DNA volume to $5 \mu$ in the first amplification. With this, thirty-two (32) were identified as
C. albicans and high (8) as C. parapsilosis, of which five (5) belong to the group II (C. orthopsilosis) and three (3) were a combination of group I and II (Figure 2).

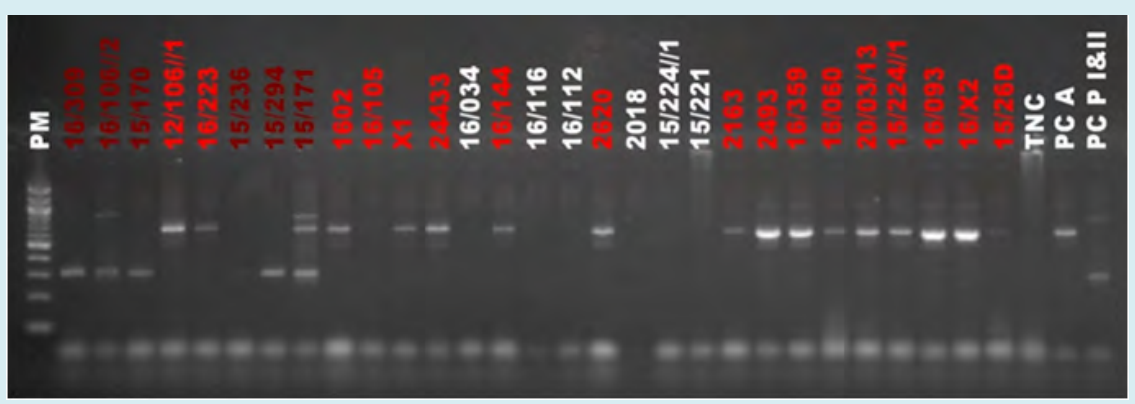

Figure 2: Multiplex PCR amplification from PsI.

PM: 100 bp molecular weight markers; TNC: Negative control; PCA: Positive control C. albicans; PCP I \& II: Positive control $C$. Parapsilosis group I and group II

With the PsII primer mix specific for $C$. dubliniensis, $C$. krusei, C. kefyr and C. glabrata, two 2 samples were positive

for C. kefyr out of 42 samples tested (Figure 3).

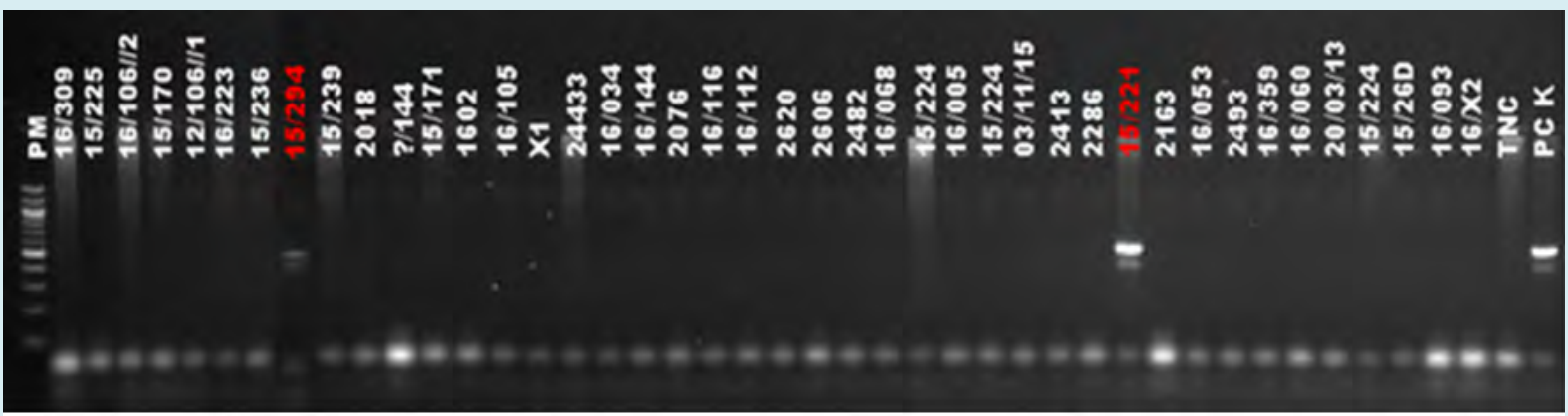

Figure 3: Multiplex PCR amplification from PsII.

PM: 100 bp molecular weight markers; TNC: Negative control; PCK: Positive control C. kefyr

PCR using PsIII, which is specific for C. tropicalis $I, C$. tropicalis II and C. lusitaniae, yielded five (5) positive samples including four (4) samples for C. tropicalis group I and one (1) for C. lusitaniae (Figure 4).

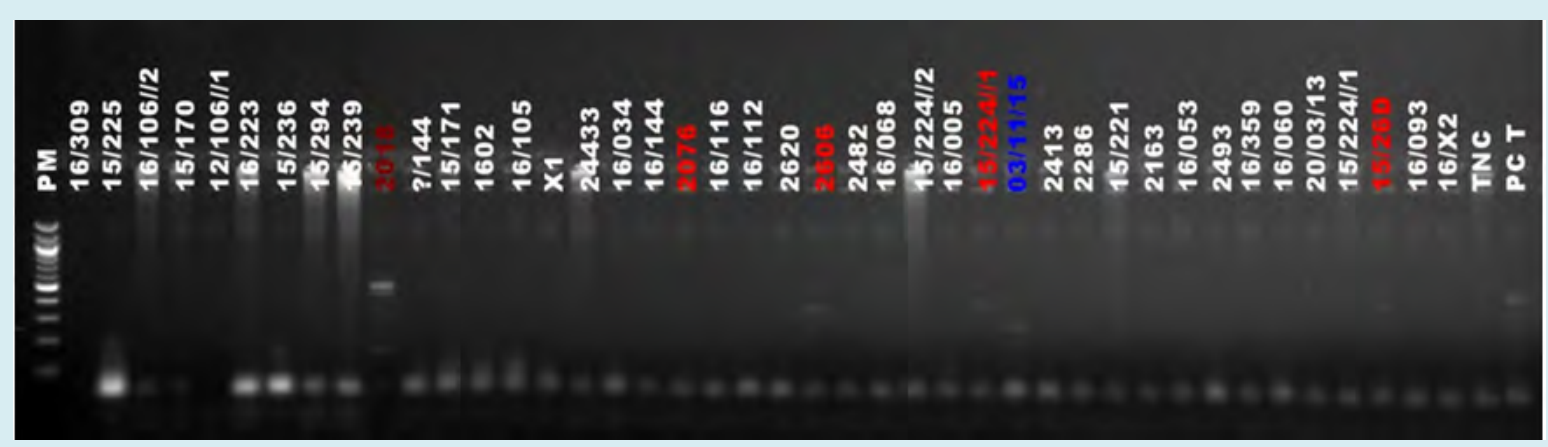

Figure 4: Multiplex PCR amplification from PsIII.

PM: 100 bp molecular weight markers; TNC: Negative control; PCA: Positive control C. tropicalis type I 
The samples negative with PSII and PSIII were tested by increasing the DNA volume, but no new positives were noted. One sample did not give results.

In total $68 \%(32 / 47)$ strains were identified as $C$. albicans; and 32\% (15/47) were non-albicans Candida. The NAC species were distributed as follow: $C$. parapsilosis I and/ or II $17 \%$ (8), C. tropicalis $8.5 \%$ (4), C. kefyr 4.25 (2) and C. lusitaniae 2.15 (1) (Figure 5).

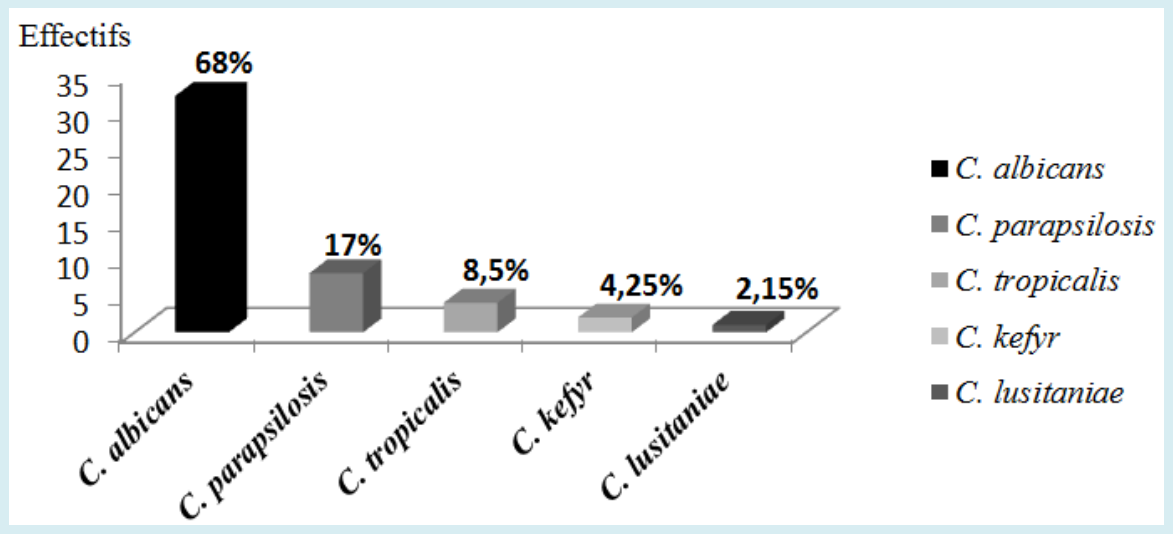

Figure 5: Frequency of the different Candida species identified.

\section{Distribution of Species According to the Origin of the Sample}

Four species of Candida were identified from skin and nail samples: $C$. albicans, $C$. parapsilosis I and the association of group I and II, C. tropicalis and $C$. kefyr. Three species were identified from vaginal samples: $C$. albicans, $C$. tropicalis and $C$. lusitaniae. Most isolates consisted of a single candida species $82.61 \%(38 / 46)$ in total (Table 2).

\begin{tabular}{|c|c|c|c|c|c|c|}
\hline Candida species & \multicolumn{2}{|c|}{ Skin \& Nail Samples (N) } & \multicolumn{2}{c|}{ Vaginal Samples (N) } & \multicolumn{2}{c|}{ Total } \\
\hline Type of infection & $\begin{array}{c}\text { Simple infection } \\
(\mathrm{N} / \%)\end{array}$ & $\begin{array}{c}\text { Mix infection } \\
(\mathrm{N} / \%)\end{array}$ & $\begin{array}{c}\text { Simple infection } \\
(\mathrm{N} / \%)\end{array}$ & $\begin{array}{c}\text { Mix infection } \\
(\mathrm{N} / \%)\end{array}$ & $\begin{array}{c}\text { Simple infection } \\
(\mathrm{N} / \%)\end{array}$ & $\begin{array}{c}\text { Mix infection } \\
(\mathrm{N} / \%)\end{array}$ \\
\hline C. albicans & $20(71.43 \%)$ & $2(33.33 \%)$ & $9(90 \%)$ & $0(0 \%)$ & $29(76.32 \%)$ & $2(25 \%)$ \\
\hline C. parapsilosis I & $4(14.29 \%)$ & $1(16.67 \%)$ & $0(0 \%)$ & $0(0 \%)$ & $4(10.53 \%)$ & $1(12.5 \%)$ \\
\hline $\begin{array}{c}\text { C. parapsilosis } \\
\text { I\& II }\end{array}$ & $2(7.142 \%)$ & $1(16.67 \%)$ & $0(0 \%)$ & $0(0 \%)$ & $2(5.26 \%)$ & $1(12.5 \%)$ \\
\hline C. tropicalis & $1(3.571 \%)$ & $1(16.67 \%)$ & $0(0 \%)$ & $2(0 \%)$ & $1(2.63 \%)$ & $3(37.5 \%)$ \\
\hline C. kefyr & $1(3.571 \%)$ & $1(16.67 \%)$ & $0(0 \%)$ & $0(0 \%)$ & $1(2.63 \%)$ & $1(12.5 \%)$ \\
\hline C. lusitaniae & $0(0 \%)$ & $0(0 \%)$ & $1(10 \%)$ & $0(0 \%)$ & $1(2.63 \%)$ & $0(0 \%)$ \\
\hline Total & $28(82.35 \%)$ & $6(17.65 \%)$ & $10(83.33 \%)$ & $2(16.67 \%)$ & $38(82.61 \%)$ & $8(17.39 \%)$ \\
\hline
\end{tabular}

Table 2: Species distribution and type of infections according to the sample origin.

\section{Comparison of Phenotypic and Molecular Identification Results}

According to the phenotypic method, $62 \%$ of the species were identified as C. albicans and $38 \%$ as NAC. With the molecular method, $68 \%$ of the species were identified as C. albicans and 32\% of NAC. Between 2008 and 2015, the distribution of candida albicans was higher than for NAC than between 2016 and 2019, respectively 80\% and 43\% (Figure $6)$. There was an increase of NAC between the two periods $31.41 \%$ (185/589) with the phenotypic method, co-infection could not be detected, whereas with the PCR $17.39 \%$ of the strains were identified as mixed with two species of Candida (Table 2). 


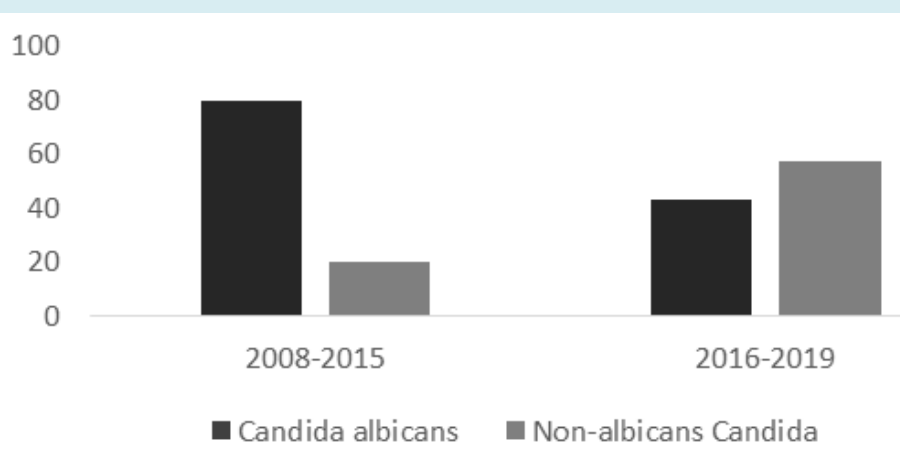

Figure 6: Distribution of Candida albicans and NAC between two time periods: 2008-2015 and 2016-2019 in Aristide LeDantec hospital, Dakar

\section{Discussion}

The objective of this study was to identify the Candida species responsible of skin, nail and vaginal infections in patients consulting the Aristide Le Dantec Hospital using nested multiplex PCR. Conventional identification by culture and blast test was previously used to diagnose the infection among those outpatients. This method only allowed identification of the C. albicans complex and the other species were reported under the name NAC. Multiplex nested PCR was then used for accurate identification and determination of the frequency of Candida species.

A total of six species of Candida were identified and are in order of frequency C. albicans, $C$. tropicalis group I, $C$. parapsilosis group I (C. parapsilosis), C. parapsilosis group II (known as C. orhtopsilosis), C. kefyr and C. lusitaniae. These results were similar to those reported from other African countries [7,9,12-14].

These results showed that, although $C$. albicans remains the most frequent species in candidiasis, other species are also incriminated [15-19] and could be more frequent if reliable identification methods such as molecular biology techniques are used. Here an increasing number of NAC were noted between these two periods showing the importance of the species identification during patient care. Unfortunately, these identification techniques are not often available in resource-limited countries. In Senegal, in most laboratories of public health structures, identification is only based on culture and blastese test, which does not allow discriminating the albicans complex from other complexes of the genus Candida.

However, this identification is essential since the sensitivity to antifungal agents can be different according to the Candida species. For instance, C. glabrata and C. krusei are resistant to triazoles drugs, particularly fluconazole, and their involvement in pathology is increasing [20]. Moreover, the increase in azole resistance of candida species requires accurate species identification [21].

The results also showed that species associations are not uncommon and has been shown by other studies [22]. In this study $17.39 \%$ of the isolates were found to be an association of species. These associations may be missed when using culture on Sabouraud's medium and the tube germ test. The determination of this association is important in the case that one of the specie is known for antifungal resistance.

The limitations of this study are the number of strains that were identified, particularly for vaginal infections for which only ten isolates were collected. It would be interesting to have isolates from deep infections to study the involvement of NAC species in those pathologies.

\section{Conclusion}

This study demonstrated that there was a diversity of Candida species responsible for superficial infections in Dakar. However, the identification of these species was not possible for these patients as the diagnosis methods available at the laboratory could not discriminate the isolates. The technical platform of developing countries must be strengthened to reliably improve the identification of microorganisms.

\section{Statements \& Declarations}

Not applicable

\section{Funding}

The work was supported by Cheikh Anta Diop University and Laboratory of Parasitology and Declarations (ethics). 


\section{Open Access Journal of Mycology \& Mycological Sciences}

\section{Conflict of Interest}

Competing interests (include appropriate disclosures): None Ethical approval was not needed the isolates used are from patient care.

\section{References}

1. Fornari G, Vicente VA, Gomes RR, Muro MD, Pinheiro RL, et al. (2016) Susceptibility and molecular characterization of Candida species from patients with vulvovaginitis. Braz J Microbiol 47(2): 373-380.

2. Sobel J (1990) Vaginal infections in adult women. Med Clin North Am 74(6): 1573-1602.

3. Develoux M, Bretagne S (2005) Candidiasis and yeast infections. EMC - Maladies Infectieuses 2(3): 119-139.

4. Diongue K, Baha Z, Seck MC, Ndiaye M, Diallo MA, et al. (2018) Cutaneous-nail candidiasis diagnosed in the parasitology and mycology laboratory of the CHU Le Dantec in Dakar from 2008 to 2015. Tropical Medicine and Health 28(4): 390-394.

5. Liu L (2013) Identification and characterization of the molecular genetic bases responsible for the predisposition to chronic cutaneous-mucous candidiasis in humans, pp : 188.

6. Mahmoudi M, Farhoomand P, Azarfar A (2012) Effects of graded levels of hemp seed (Cannabis sativa L.) on performance, organ weight and serum cholesterol levels on broilers. J Medic Plants 2(42): 121-129.

7. Sylla K, Tine RCK, Sow D, Lelo S, Dia M, et al. (2019) Epidemiological and Mycological Aspects of Onychomycosis in Dakar (Senegal). J Fungi (Basel) 5(2): 35.

8. Seyoum A, Bitew A, Mihret A (2020) Distribution of Candida albicans and non albicans Candida species isolated in different clinical samples and their in vitro antifungal suscetibity profile in Ethiopia. BMC Infect Dis 20(1): 231.

9. Ogbolu DO, Oni AA, Daini OA, Oloko AP (2007) In vitro antimicrobial properties of coconut oil on Candida species in Ibadan, Nigeria. J Med Food 10(2): 384-387.

10. Kanbe T, Horii T, Arishima T, Ozeki M, Kikuchi A (2002) PCR-based identification of pathogenic Candida species using primer mixes specific to Candida DNA topoisomerase II genes. Yeast 19(11): 973-989.

11. Kato H, Kato N, Watanabe K, Yamamoto T, Suzuki K, et al. (2001) Analysis of Clostridium difficile isolates from nosocomial outbreaks at three hospitals in diverse areas of Japan. J Clin Microbiol 39(4): 1391-1395.

12. Letscher-Bru V (2003) Candidoses. Enc Meed Chir Biologie Medicale, Paris: Elsevier Masson SAS, pp: 1-22.

13. Poulain D (2013) Candida albicans, plasticité et pathogénie. Rev Fr Lab 450: 37-46.

14. Seyoum A, Bitew A, Mihret A (2020) Distribution of Candida albicans and non albicans Candida species isolated in different clinical samples and their in vitro antifungal suscetibity profile in Ethiopia. BMC Infect Dis 20(1): 231.

15. Kwamin F, Nartey NO, Codjoe FS, Newman MJ (2013) Distribution of Candida species among HIV-positive patients with oropharyngeal candidiasis in Accra, Ghana. J Infect Dev Ctries 7(1): 41-45.

16. Ngouana TK, Toghueo RMK, Kenfack IF, Lachaud L, Nana AK, et al. (2019) Epidemiology and antifungal susceptibility testing of non-albicans Candida species colonizing mucosae of HIV-infected patients in Yaoundé (Cameroon). J Mycol Med 29(3): 233-238.

17. Snydman DR (2003) Shifting in patterns in the epidemiology of nosocomial Candida infections. Chest 123(5 S): 500-503.

18. Sobel JD (2006) The emergence of non-albicans Candida species as causes of invasive candidiasis and candidemia. Curr Infct Dis Rep 8(6): 427-433.

19. Latha R, Sasikala R, Muruganandam RN, Babu RY (2011) Study on the shifting patterns of Non Candida albicans Candida in lower respiratory tract infections and evaluation of the CHROM agar in identification of the Candida species. J Microbiol Biotech Res 1: 113-119.

20. Alcazar-Fuoli L, Mellado E (2014) Current status of antifungal resistance and its impact on clinical practice. Br J Haematol 166(4): 471-484.

21. Pfaller MA, Diekema DJ, Messer SA, Boyken L, Hollis RJ (2003) Activities of fluconazole and voriconazole against 1,586 recent clinical isolates of Candida species determined by Broth microdilution, disk diffusion, and Etest methods: report from the ARTEMIS Global Antifungal Susceptibility program, 2001. J Clin Microbiol 41(4): 1440-1446.

22. Agwu E, Ihongbe JC, McManus BA, Moran GP, Coleman DC, et al. (2012) Distribution of yeast species associated with oral lesions in HIV-infected patients in Southwest Uganda. Med Mycol 50(3): 276-280.

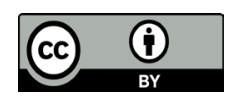

